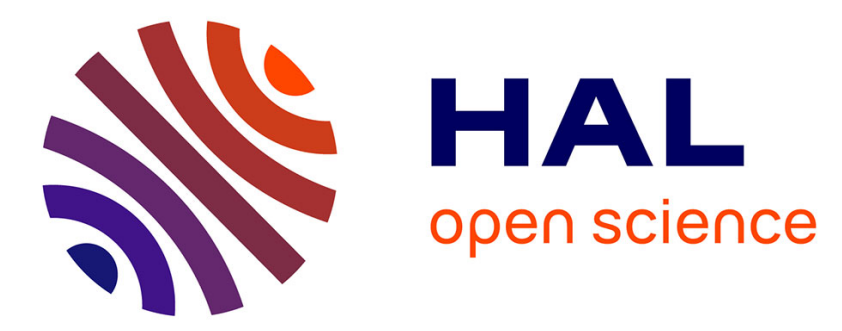

\title{
Perspectives ethnologiques dans les organisations modernes
}

Denis Guigo

\section{To cite this version:}

Denis Guigo. Perspectives ethnologiques dans les organisations modernes. L'Homme - Revue française d'anthropologie, 1992, 32 (121 (Anthropologie du proche, ss la direction de Marc Abélès et Susan Rogers)), pp.47-65. halshs-00067751

\section{HAL Id: halshs-00067751 https://shs.hal.science/halshs-00067751}

Submitted on 1 Apr 2010

HAL is a multi-disciplinary open access archive for the deposit and dissemination of scientific research documents, whether they are published or not. The documents may come from teaching and research institutions in France or abroad, or from public or private research centers.
L'archive ouverte pluridisciplinaire HAL, est destinée au dépôt et à la diffusion de documents scientifiques de niveau recherche, publiés ou non, émanant des établissements d'enseignement et de recherche français ou étrangers, des laboratoires publics ou privés. 


\section{Persée}

http://www.persee.fr

\section{Perspectives ethnologiques dans les organisations modernes}

\section{Denis Guigo}

Guigo Denis, . Perspectives ethnologiques dans les organisations modernes. In: L'Homme, 1992, tome $32 \mathrm{n}^{\circ} 121$.

Anthropologie du proche. pp. 47-65.

Voir l'article en ligne

\section{Avertissement}

L'éditeur du site «PERSEE » - le Ministère de la jeunesse, de l'éducation nationale et de la recherche, Direction de l'enseignement supérieur, Sous-direction des bibliothèques et de la documentation - détient la propriété intellectuelle et les droits d'exploitation. A ce titre il est titulaire des droits d'auteur et du droit sui generis du producteur de bases de données sur ce site conformément à la loi n`98-536 du 1 er juillet 1998 relative aux bases de données.

Les oeuvres reproduites sur le site «PERSEE » sont protégées par les dispositions générales du Code de la propriété intellectuelle.

Droits et devoirs des utilisateurs

Pour un usage strictement privé, la simple reproduction du contenu de ce site est libre.

Pour un usage scientifique ou pédagogique, à des fins de recherches, d'enseignement ou de communication excluant toute exploitation commerciale, la reproduction et la communication au public du contenu de ce site sont autorisées, sous réserve que celles-ci servent d'illustration, ne soient pas substantielles et ne soient pas expressément limitées (plans ou photographies). La mention Le Ministère de la jeunesse, de l'éducation nationale et de la recherche, Direction de l'enseignement supérieur, Sous-direction des bibliothèques et de la documentation sur chaque reproduction tirée du site est obligatoire ainsi que le nom de la revue et- lorsqu'ils sont indiqués - le nom de l'auteur et la référence du document reproduit.

Toute autre reproduction ou communication au public, intégrale ou substantielle du contenu de ce site, par quelque procédé que ce soit, de l'éditeur original de l'oeuvre, de l'auteur et de ses ayants droit.

La reproduction et l'exploitation des photographies et des plans, y compris à des fins commerciales, doivent être autorisés par l'éditeur du site, Le Ministère de la jeunesse, de l'éducation nationale et de la recherche, Direction de l'enseignement supérieur, Sous-direction des bibliothèques et de la documentation (voir http://www.sup.adc.education.fr/bib/ ). La source et les crédits devront toujours être mentionnés. 


\section{Perspectives ethnologiques dans les organisations modernes}

Denis Gulgo, Perspectives ethnologiques dans les organisations modernes. - A partir d'enquêtes effectuées dans une entreprise automobile française et dans une mairie argentine, ce texte propose une caractérisation ethnologique de l'activité humaine dans les grandes organisations modernes. Une entreprise ou une administration pose pour ses membres un cadre spécifique d'activité (locaux, instruments de travail, statut et segmentation des agents, hiérarchie...) ; ces logiques de différenciation du personnel et de répartition des tâches sont en principe gouvernées par la correspondance des fins et des moyens ; cette relative transcendance des finalités officielles donne un fondement à l'exercice de l'autorité. Toutefois, les "êtres totaux " que sont les membres de l'organisation peuvent aussi y mobiliser d'autres facettes de leur identité ; ils réalisent en définitive un " bricolage " imaginatif à partir des éléments organisationnels - matériels ou symboliques - qui sont à leur portée ; on relève l'importance des pratiques de mobilisation du symbolique et de l'imaginaire, mises en œuvre par des membres investis ou non de l'autorité officielle. Les grandes organisations apparaissent ainsi comme un terrain prometteur pour l'investigation ethnologique ; comme dans l'étude des objets plus " traditionnels ", il ne s'agit pas d'appréhender la totalité de la société, mais " d'y discerner des niveaux qui soient comparables, et deviennent ainsi significatifs " (LéviStrauss $1958: 100)$.

Les grandes organisations de la société moderne (entreprises, administrations, associations...) offrent-elles un terrain propice à l'exercice de l'ethnologie ? A moins de se cantonner dans un atelier ou dans un bureau, on y rencontre rarement l'authenticité qui caractérisait naguère, selon Claude Lévi-Strauss, les sociétés étudiées par les ethnologues : celles où les relations sociales sont fondées sur l' " appréhension concrète d'un sujet par un autre ", et non sur " toutes sortes d'intermédiaires - documents écrits ou mécanismes administratifs $»^{1}$.

Toutefois, la diversification des thèmes abordés par les ethnologues dans les sociétés modernes, principalement à partir des années 70 , a rendu caduc le critère de l'authenticité ${ }^{2}$. La spécificité de l'ethnologie réside plus dans son approche - le " regard éloigné ", qui en fait " l'essence et l'originalité ${ }^{3}-$ que dans le type de société appréhendée. L'altérité de l'ethnologue est en fait 
un instrument, dont il joue pour relativiser ses préjugés et se présenter sur son terrain d'enquête. Il réduit sa distance en cherchant à se situer au plus près de ceux qu'il étudie ; il la reconstitue au moment de procéder à l'analyse et à la comparaison. L'observateur peut appliquer cette dynamique de distanciation $^{4}$ à un groupe de sa propre société, à condition de se faire, suivant l'expression d'André Leroi-Gourhan, " comme étranger à son propre milieu $»^{5}$. Dans ce va-et-vient entre identification subjective et analyse objective, " l'ethnologie rapprochée " rejoint donc " l'ethnologie lointaine ${ }^{6}$.

Si les démarches sont analogues, les points de départ sont différents : dans une société " exotique ", l'étrangeté de l'observateur est immédiatement donnée, tandis que chez nous, la distance est d'abord à construire ${ }^{7}$. Encore faut-il préciser ce qu'étudie cette ethnologie de la modernité, et se prémunir contre le risque « de confondre l'objet empirique et l'objet intellectuel $»^{8}$. Les entreprises, les administrations et autres entités de la société industrielle peuvent certes être rassemblées sous le vocable général d'organisations, mais cette catégorisation du sens commun ne suffit pas à poser l'organisation comme objet ethnologique. En revanche, le groupe formé par les membres d'une organisation entre bien dans le champ de l'ethnologie : « la considération d'individus constituant des groupes qui se perçoivent ou sont perçus comme des unités distinctes ${ }^{9}$. $\mathrm{Ce}$ critère identitaire s'applique souvent dans l'entreprise : "Vous êtes de la maison ? ", demande-t-on à une tête nouvelle. Les membres d'une organisation sont identifiés et distingués, rémunérés, parfois vêtus ; ils se voient assigner des tâches à réaliser dans des lieux et des temps déterminés. Des dynamiques de différenciation s'exercent ainsi à plusieurs niveaux : en particulier, la division des tâches et la hiérarchie fondent des catégorisations " horizontales » et " verticales » internes, dont certaines s'emboîtent les unes dans les autres. Identification, différenciation, segmentation : voilà qui intéresse au premier chef l'ethnologie.

L'ethnologie industrielle s'est pourtant développée tardivement, en France en particulier. Les premières enquêtes ethnographiques en usine furent réalisées aux États-Unis à la fin des années 20, dans une optique d'anthropologie appliquée qui donna naissance au courant réformiste dit " human relations school ", qui ambitionnait de corriger "l'inhumanité " du système industriel taylorien ${ }^{10}$. On ne relatera pas ici l'histoire de l'anthropologie industrielle ; signalons simplement que les travaux ultérieurs se partagèrent principalement entre des perspectives réformistes, qui recherchaient un fonctionnement plus harmonieux des organisations, des perspectives critiques, qui visaient à remettre en question le système capitaliste, et un certain nombre d'études comparatives (« crosscultural »), qui s'intéressaient à des aspects fort divers de l'organisation industrielle ${ }^{11}$. Plus récemment, plusieurs programmes de recherche ont été consacrés au recueil de patrimoines en cours de disparition (savoir-faire et milieux industriels en déclin ou en reconversion) ${ }^{12}$; assez peu nombreuses ont été les enquêtes ethnographiques menées sur des terrains industriels non menacés d'obsolescence, qu'on pourrait dire "centraux ", au sens où Marc Abélès parle 
d'anthropologie des centres politiques et administratifs dans sa contribution à ce numéro ${ }^{13}$. L'ampleur du champ de recherche potentiel, à l'heure où les grandes organisations sont omniprésentes dans le paysage changeant de la société moderne, devrait inciter à multiplier les études des milieux (post)-industriels.

Il est bien sûr trop tôt pour tirer des conclusions générales à partir des matériaux disponibles ; toutefois, au lieu de se satisfaire de l'accumulation de monographies décrivant des terrains souvent fort différents les uns des autres, il ne paraît pas prématuré d'esquisser, à la lumière de certains premiers résultats, quelques fils conducteurs qui semblent particulièrement prometteurs pour l'anthropologie des hommes des grandes organisations modernes. L'ambition de cet article est de poser des jalons dans cette direction, à partir de deux enquêtes de terrain réalisées en 1985 dans une entreprise française et en 1987 dans une mairie argentine.

Examinons tout d'abord le cas d'une mairie de la banlieue de Buenos Aires, qui administre un département (partido) de $200 \mathrm{~km}^{2}$, où résident environ 700000 habitants $^{14}$. Le maire et le conseil municipal sont élus par les citoyens du département; les attributions de la municipalité, définies par une loi provinciale ${ }^{15}$, concernent principalement l'aménagement du territoire municipal, les normes d'édification, l'installation des industries et des commerces, le tracé et l'entretien des rues, les activités culturelles et sportives, les hôpitaux municipaux, les cimetières, ainsi que les services publics de nettoiement, d'eau, d'éclairage public et de transport (sauf quand ils sont assurés par un autre organisme, comme c'est le cas pour l'électricité par exemple).

Lors de mes premiers contacts avec la mairie, je ne recherchais que des informations statistiques générales sur ce département, afin de compléter une enquête portant sur la compagnie d'électricité du Grand Buenos Aires. Toutefois, la chaleur de l'accueil de mes premiers interlocuteurs, ainsi que les dynamiques complexes du palacio, le siège du gouvernement municipal, m'incitèrent à prendre la mairie comme objet de recherche. Je ne présentai pas de demande d'étude aux autorités municipales, car la proximité des élections de septembre 1987 - pour lesquelles le fils du maire était donné gagnant - provoquait de vives controverses sur l'identité politique des divers courants du parti au pouvoir (le parti péroniste ${ }^{16}$ ), ainsi que sur la loyauté ou la traîtrise des différents responsables. Si j'envisageais de prendre pour objet d'étude ces logiques de différenciation, je tenais à rester extérieur aux enjeux de pouvoir locaux.

Il semble difficile d'enquêter dans une entreprise sans l'autorisation de la direction. En revanche, l'accès à une mairie est plus aisé, ne serait-ce que parce que les autorités d'une municipalité en représentent les habitants et administrent certains aspects de leur vie quotidienne. Du bureau du cadastre aux services fiscaux, de la salle du conseil aux permanences des élus, il y a mille prétextes pour flâner dans une mairie. Les bureaux municipaux ne sont pas pour autant 
des lieux publics. La collaboration effective de mes interlocuteurs dépendait de l'instauration d'une connivence entre nous, créée par le partage d'une base de connaissances sur l'histoire municipale, d'une part, et de l'intérêt porté aux questions spécifiques relevant de ceux avec qui je prenais langue, d'autre part. Cette relative communauté intellectuelle et affective nous permettait d'aborder ensuite une grande diversité de thèmes. Tirant parti du caractère semi-ouvert du palais municipal, je réalisai ainsi de multiples entretiens au fil des réseaux d'affinité.

3140 agents travaillaient à la mairie (dont un tiers dans le palais proprement dit) : en haut de l'échelle hiérarchique, une quarantaine de "fonctionnaires de direction ", alias " fonctionnaires politiques "; sous leurs ordres, environ trois mille " agents permanents » (qui bénéficiaient, eux, de la stabilité de l'emploi) ; entre ces deux catégories, une centaine de "coordinateurs ", qui étaient en quelque sorte les commissaires politiques de l'organisation, car le maire les avait mis en place pour contrôler de plus près les fonctionnaires de carrière et rétribuer l'engagement politique de certains militants. A l'exception des coordinateurs, tout le personnel était classé dans l'une des catégories de " l'échelle mobile municipale » (de 2 à 25), qui définissait la hiérarchie des agents et leur rémunération ${ }^{17}$.

Les agents municipaux étaient jugés avant tout sur leur ponctualité. Arriver plus de deux minutes après l'heure réglementaire ( $7 \mathrm{~h}$ en été, $8 \mathrm{~h}$ en hiver) était considéré comme un retard; trois retards dans le mois entraînaient en principe un jour de renvoi sans salaire. Une fois installé à sa place, chacun devait en principe se consacrer à ses fonctions, sous peine d'encourir les sévères sanctions prévues par le statut du personnel. Cependant, durant ses six heures de présence, l'agent pouvait recevoir un certain nombre de prestations annexes : loteries, achats d'habillement et d'objets divers (vêtements pour adultes ou pour enfants, chaussures, draps, parfumerie, bijouterie...). Les échantillons étaient exposés dans la petite cuisine de chaque étage et les toilettes servaient de salon d'essayage. On pouvait aussi bénéficier d'un certain nombre de services personnels : se faire couper les cheveux par une employée dans la cuisine, voire s'y faire arracher une dent par un dentiste ambulant. Le temps des employés, strictement contrôlé en théorie, se prêtait en réalité à bon nombre d'aménagements. De même, l'ethnographie d'un service municipal, brièvement présentée ci-dessous, a montré que les agents pouvaient utiliser, d'une manière particulièrement imaginative, les éléments - matériels ou symboliques - de l'organisation.

Le service en question est responsable de l'urbanisme municipal ; ses membres occupent au total $50 \mathrm{~m}^{2}$ de bureaux : le directeur, sa secrétaire, quatre professionnels (diplômés d'études supérieures) et sept employé(e)s chargés de les assister. Toutefois, à la mi-87, le personnel était en fait divisé en deux groupes, que j'appellerai ici le groupe de la secrétaire et le groupe des professionnels. Quelle que soit la tâche à remplir, toute collaboration était pratiquement interrompue entre les uns et les autres. Les protagonistes étaient approximativement groupés par affinités dans l'espace du service. La secrétaire du directeur pouvait compter 
sur l'aide de trois employés masculins et de la cartographe. Le groupe des professionnels était appuyé par trois employées. En cas de conflit entre les deux groupes, le directeur ne tranchait jamais en défaveur de sa secrétaire. Cette dernière contrôlait d'autre part l'administration des éléments de base de la vie du bureau : outils de travail (papier, crayons, machines à écrire, meubles...), instruments de contrôle du personnel (feuilles de présence quotidiennes, demandes de congé...), communication des informations internes.

Tous les objets du bureau, loin d'être de simples outils fonctionnels attribués selon les tâches de chacun, étaient utilisés en fonction du conflit qui divisait la direction. Par exemple, il était rare qu'un membre du groupe des professionnels obtienne plus d'une feuille de papier ou d'une chemise à la fois. S'il demandait du papier à en-tête : " Il n'y en a pas, il n'y en a pas ! " En réalité, il était dissimulé sous le papier blanc. Les appels téléphoniques étaient également administrés au compte-gouttes. Une professionnelle n'en reçut pratiquement aucun pendant plusieurs mois : ils se " perdaient " au standard ou étaient coupés à l'arrivée, d'un discret mouvement de doigt.

Les actes quotidiens de la vie du bureau conduisaient les agents à se regrouper à tel ou tel titre : les membres de la direction, ceux qui partageaient le café, les professionnels du service, etc. L'appartenance à ces différents cercles constituait l'enjeu et l'instrument de nombreuses stratégies internes. La secrétaire ourdit ainsi l'exclusion d'une professionnelle du partage du café matinal, en ordonnant aux employés - qui le préparaient car ils arrivaient les premiers de ne pas lui en laisser une seule goutte afin de châtier son arrogance supposée («Qu'est-ce qu'elle se croit ? Parce qu'elle est professionnelle, elle est plus que nous »?). Le café restant était jeté à la cuisine de l'étage ou versé discrètement dans des tasses vides, dans un placard. Celle-ci contre-attaqua en fondant un deuxième cercle de café et en en proposant régulièrement aux autres membres du service. Mais la petite cafetière qu'elle avait achetée à cet effet souffrit une sorte de profanation : le camp adverse l'utilisa pour faire bouillir des saucisses.

Les informations internes pouvaient aussi être manipulées : elles « s'évaporaient " parfois entre deux services. Le " dossier égaré " était d'ailleurs un syndrome classique du palais municipal. Que le fourvoiement fût accidentel ou intentionnel, le temps nécessaire à la localisation ou à la reconstitution du dossier était autant de gagné par ceux qui avaient intérêt à retarder l'affaire, à moins que la paralysie n'eût d'autre but que l'obtention d'un pot-de-vin en échange du déblocage. Certaines méthodes d'élimination des documents gênants étaient plus expéditives : quelques poutres noircies constituaient le seul vestige des archives municipales, qui avaient été ravagées par un incendie qu'on avait dit intentionnel.

La classification des agents municipaux selon leur rang dans « l'échelle mobile " était le principal outil symbolique du palais. Le passage à une catégorie supérieure, l'incorporation à un sous-groupe plus avantageux constituaient des enjeux en termes de prestige et d'obtention de moyens d'action, car cette 
différenciation commandait la répartition des moyens matériels. Dans le service de l'urbanisme, la secrétaire était classée dans une catégorie de " personnel hiérarchique » (intermédiaire entre celles des employés et celles des professionnels) ; elle tentait d'asseoir la cohésion de son groupe en contestant les capacités des professionnels et le bien-fondé des avantages que le statut du personnel leur octroyait. Notons que le directeur invitait souvent la secrétaire à des réunions techniques, affirmant qu'elle avait « le même niveau que les professionnels ». Il avait essayé par ailleurs de mettre une professionnelle de son côté en lui proposant un appartement dans un programme municipal de logement (bien qu'elle n'en remplît pas les conditions d'attribution) ; le refus de l'intéressée avait déclenché une campagne de surveillance relayée par un réseau d'alliés répartis dans l'organisation : contrôle horaire renforcé, contrôle des congés de maladie, curieux incidents administratifs, etc.

Un autre élément signalait la lutte feutrée que se livraient les deux factions du service : au plafond d'une pièce partagée par trois professionnels, une croix était disposée à l'horizontale, dans une ouverture ménagée par le retrait d'une dalle du faux plafond. Les occupants de la pièce en question supposaient que le groupe de la secrétaire avait suspendu, pour leur nuire, cette " croix inversée " au-dessus de leurs têtes. Le traditionnel symbole de protection devenait pour eux une menace. Dans un pays où crucifix et images pieuses ornaient les murs de nombreux lieux publics, où les autorités proclamaient leur allégeance aux valeurs chrétiennes, où il n'y avait pas de cérémonie publique sans un représentant de l'Église catholique - et un militaire - c'était pour le moins inquiétant. Faute de trouver la clé de certains problèmes du service (agressivité des collègues, retards dans les circuits administratifs), les professionnels les attribuaient parfois à la croix inversée, tout en affectant de ne pas la prendre au sérieux.

Nul n'y toucha pendant plusieurs mois, jusqu'au jour où le directeur, pris d'un soudain malaise, s'évanouit dans les bras d'un professionnel, qui revenait justement d'une veillée funèbre. Un conseiller municipal venu à leur aide tomba à son tour, victime d'une hémorragie nasale. Deux personnes à l'hôpital et une flaque de sang sur le plancher du bureau : il fallait réagir. Le lendemain, une professionnelle fit choir la croix en la frappant avec un rouleau de plans du département, puis la piétina énergiquement. Elle constata qu'il s'agissait d'un authentique crucifix, muni de son numéro à l'inventaire municipal, tout comme les autres meubles du palais. Elle alla alors consulter le curé, autorité légitime en la matière, qui murmura : "L'esprit humain est très complexe... " et lui remit un flacon d'eau bénite pour exorciser la croix. Des cônes d'encens furent brûlés dans le bureau des professionnels et la croix fut placée bien en évidence sur le mur du fond. Une cérémonie eut lieu dix jours plus tard : trois professionnels récitèrent un Notre Père et aspergèrent la croix d'eau bénite.

La secrétaire installa ensuite la croix sur la cloison voisine de son bureau et y colla la légende : " Père, pardonne-leur, ils ne savent pas ce qu'ils font. " La croix ayant plus tard mystérieusement disparu, elle en dessina une au même 
endroit, en y adjoignant la même prière et un ruban aux couleurs d'une « coopérative de logement " qui avait lancé plusieurs projets immobiliers sur le territoire du département. La promotion de cette association constituait justement la nouvelle croisade à laquelle le directeur exhortait le personnel (une grave pénurie de logements affectait la région); il ne pouvait toutefois compter que sur le groupe de la secrétaire, car les professionnels doutaient du sérieux de la coopérative en question.

A l'époque, le directeur participait activement à la campagne électorale ; il avait construit lui-même dans le hall du palais un bel obélisque en bois d'environ deux mètres de haut, dont les flancs célébraient les réalisations du gouvernement sortant. Le monument évoquait les obélisques qui ornent certaines places argentines en l'honneur de la fondation de la ville ou de l'indépendance nationale. Une autre touche de patriotisme figurait au mur du bureau du directeur : un " diplôme de reconnaissance » octroyé par la coopérative de logement, dont le cadre était orné de bandes aux couleurs du drapeau argentin et à celles de la coopérative. Le service de l'urbanisme avait aussi sa propre bannière, une synthèse patriotico-religieuse : une croix aux couleurs du drapeau argentin, avec une petite maison au centre. Remarquons enfin que la coopérative avait organisé, le jour de la Fête nationale du drapeau, une " cérémonie civique de pose de la pierre fondamentale " pour inaugurer l'un de ses projets de construction. Les opposants au directeur, à sa secrétaire ou à la coopérative qu'ils parrainaient se trouvaient ainsi symboliquement rejetés dans une antipatrie ${ }^{18}$ sacrilège.

Certains employés jugèrent que la professionnelle qui avait fait choir le crucifix avait accompli une prouesse risquée. En effet, la capacité de la secrétaire à gagner des alliés et à se faire obéir des employés se nourrissait d'une croyance répandue dans le palais, selon laquelle elle avait l'oreille de deux institutions - l'Église catholique et les services de renseignement militaires - ainsi que des hautes autorités municipales. Le soutien que la secrétaire était censée avoir des autorités était sans doute imaginaire, mais cela n'empêchait pas son dispositif d'être opératoire. Ses collègues de travail en percevaient les effets sur les objets du bureau, les corps et les esprits. Certains croyaient à ses appuis terrestres, d'autres la considéraient comme une possédée : "C'est l'incarnation du mal "; " elle a le diable au corps ». Son influence jouait sans doute aussi sur un autre registre : c'était une femme encore jeune, aux yeux bleus, jugée attirante ; tous les employés masculins de la direction s'étaient justement rangés à ses côtés. En définitive, son ascendant faisait appel au symbolique et à l'imaginaire, les fondements universels du pouvoir : « Le pouvoir, partout et tou-

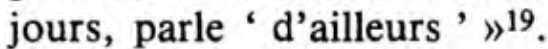

Outre la mise en jeu de diverses légitimités - consubstantielles ou non à l'institution municipale - et l'articulation de réseaux d'alliés internes, l'exercice du pouvoir au sein du service faisait largement appel à la manipulation des composantes de l'organisation ${ }^{20}$. La secrétaire du directeur, en particulier, soumettait ses collègues à un ensemble de petites techniques de contrôle, dont les effets se cumulaient dans l'espace sans fenêtre et le temps répété du bureau. 
La plupart des instruments de bureau étaient traités comme les munitions de cette " technologie des pouvoirs subtils » ${ }^{21}$, qui combinait éléments matériels et aspects symboliques, en jouant sur les rouages organisationnels et sur la différenciation des individus ${ }^{22}$.

Après l'ethnographie d'un service municipal, quelle vision ethnologique de l'organisation peut-on proposer ? Une étude globale du palais municipal a certes été effectuée à l'occasion de cette recherche (données historiques et politicoéconomiques, fonctionnement des services et gestion du personnel, utilisation du budget, dynamiques syndicales...), mais ce n'est pas d'une monographie générale qu'il s'agit ici ${ }^{23}$. Remarquons tout d'abord que, pour pouvoir interpréter les enjeux du bureau, nous avons dû prendre en compte bon nombre d'éléments qui concernaient l'ensemble de la mairie ou qui renvoyaient à des sphères de légitimité extra-institutionnelles. Le service examiné n'était pas un monde clos, mais un espace situé dans un contexte organisationnel et où les protagonistes, en tant qu' " êtres totaux ", pouvaient mobiliser des facettes extérieures de leur identité.

On relevait d'autre part, à différents niveaux de l'institution municipale, des fonctionnements qui " résonnaient en phase " avec les dynamiques observées dans le service étudié. On a vu que les agents se taillaient largement leur propre temps au sein de l'organisation. Certains utilisaient également les ressources de la mairie à diverses fins (tirage de plans, photocopies, recherche de clients pour des activités privées...) ; les autorités du palais, quant à elles, n'hésitaient pas à faire de même au bénéfice du parti péroniste (embauche massive de militants, préparation des campagnes électorales). L'échelle mobile, épine dorsale de l'organisation, semblait elle-même éminemment flexible : pour satisfaire les revendications syndicales ou favoriser telle ou telle catégorie d'agents, on modifiait fréquemment la catégorisation ou on mettait en place des rémunérations additionnelles. Cette plasticité des instruments de gestion, jointe aux nombreuses possibilités de manipulation et de stratégie, plaçait la vie au palais sous le signe dynamique d'un bricolage imaginatif. Les composantes de l'organisation - données à ses membres ou construites par eux constituaient les matériaux de leur activité créatrice et formaient de puissants outils qui étaient loin d'être réservés aux autorités officielles.

Remarquons enfin que la décoration du bureau du maire, tout comme les soutiens dont était créditée la secrétaire du service de l'urbanisme, se basait sur une triade politico-ecclésiastico-sécuritaire : crucifix, drapeau, photos et bustes de célébrités politiques, objets offerts par les autorités religieuses ou policières... Il en était généralement de même pour les autres responsables municipaux. Nous avons noté la présence de représentants de l'Église et des Forces armées à toutes les cérémonies officielles. Les membres du palais étaient ainsi conduits à prendre en compte, outre les normes et hiérarchies municipales, diverses institutions qui auraient été difficiles à invoquer explicitement, mais qui étaient mobilisées par des pratiques symboliques ou en en appelant à l'imaginaire des agents. 
En définitive, travailler au palais, c'était prendre en compte les différents réseaux administratifs, politiques, syndicaux, religieux et familiaux ; en subir les feux croisés ou bâtir, grâce à leur articulation, des stratégies élaborées ${ }^{24}$. L'ethnographie d'un service a donc dégagé des fils conducteurs pertinents pour l'appréhension globale de l'activité des agents municipaux.

Penchons-nous maintenant sur une tout autre société : une entreprise française de construction automobile. L'analyse de l'activité de ses membres va-t-elle rejoindre les perspectives esquissées dans l'étude de la mairie ?

L'entreprise en question a son siège social à Paris ; ses usines et ses bureaux d'études sont disséminés dans plusieurs régions françaises. Fin 1984, la direction du personnel me demanda un rapport sur la mise en œuvre du management participatif dans les usines ${ }^{25}$. En effet, depuis le début des années 80 , un grand nombre d'innovations de gestion avaient été expérimentées dans les services les plus divers, sous le signe d'une plus active participation de toutes les catégories d'agents - ceux «d'en bas » comme ceux "d'en haut » - et la direction du personnel souhaitait confier à un œil extérieur un bilan des initiatives locales. La plus visible de ces innovations, le « cercle de qualité ", proposait à des groupes d'une dizaine de volontaires - ouvriers, chefs d'équipe et techniciens mêlés - de résoudre d'une manière plus participative certains problèmes techniques ou organisationnels qui les concernaient directement (éclairage des locaux, disposition des machines, qualité des produits...).

Les dits cercles ne constituaient que la partie émergée d'un mouvement plus profond : dans la plupart des usines, certains chefs de service mettaient en place des groupes de travail où tous étaient invités à s'exprimer. Même si cette entorse à la taylorisation régnante ne concernait que des thèmes définis de manière " constructive » par la direction (sécurité, conditions de travail, productivité...), il n'en restait pas moins que les agents subalternes se voyaient considérés, souvent pour la première fois, comme sujets pensants, capables d'émettre une opinion sur le contenu et l'organisation du travail.

On voit qu'il n'était nullement question de subvertir la pyramide hiérarchique ; bien au contraire, la direction veillait à définir strictement le cadre de ces groupes et à réaffirmer son rôle lors des cérémonies de clôture. Un directeur déclarait ainsi dans une interview : " J'ai décidé de mettre en place une démarche participative. " Il ne s'agissait pas non plus de supprimer la distinction entre concepteurs et exécutants ; simplement, ces derniers étaient invités à donner leur avis. Un faisceau de facteurs généraux encourageait cette ouverture prudente : la plus forte réticence des nouvelles générations à être traitées comme des rouages anonymes ; une crise sévère qui avait fait plonger les comptes de l'entreprise « dans le rouge " ; enfin, les craintes de l'encadrement suite à la promulgation des lois Auroux sur les "libertés des travailleurs ", qui évoquaient pour de nombreux cadres le fantasme des soviets dans l'usine et les poussaient à prendre l'initiative. 
Les éléments que nous allons présenter n'ont pas trait aux cercles de qualité : malgré leur grande importance symbolique, les cercles laissent quasiment inchangées les tâches quotidiennes et ne concernent jamais qu'une minorité du personnel. En revanche, au cœur des usines de fabrication, de nouvelles pratiques de gestion, axées sur l'autocontrôle, entraînent des évolutions notables pour tout le personnel. Trois ateliers de fabrication des mêmes modèles de véhicules, dans trois usines françaises, ont été comparés : examinons le cas de l'un d'entre eux, où l'autocontrôle était alors en cours d'instauration.

Il s'agit d'ateliers de "montage " : à la sortie des installations de peinture robotisées, c'est l'étape où la voiture prend corps. La plupart des pièces moteurs et sièges exceptés - y sont montées sur les carrosseries. Il y a peu de machines automatiques et les outils des ouvriers " monteurs " ne sont guère plus sophistiqués que des visseuses. Le travail se fait " à la chaîne "; les ouvriers doivent donc accomplir leurs tâches en accompagnant le lent mais inexorable déplacement d'une crémaillère qui emmène les " caisses " à assembler d'une extrémité à l'autre du bâtiment. Le temps d'intervention des monteurs sur chaque voiture est de l'ordre de trois minutes, à l'issue desquelles ils reviennent une dizaine de mètres en arrière pour reprendre les mêmes opérations sur le véhicule suivant.

Dans cet atelier, chaque chaîne rassemble une centaine d'ouvriers ; il y avait autrefois une dizaine de contrôleurs et une dizaine de retoucheurs en bout de chaîne. Aujourd'hui, il subsiste deux ou trois retoucheurs pour chaque chaîne et il n'y a plus de contrôleurs : ce sont les monteurs eux-mêmes qui signalent, sur un formulaire, les opérations qu'ils n'ont pu mener à bien. Un chef d'équipe, de statut maîtrise (la catégorie intermédiaire entre les ouvriers et les cadres), dirige une quinzaine d'ouvriers. Chaque chef d'équipe est assisté d'un " moniteur ", qui est de statut ouvrier mais a l'aspect d'un agent de maîtrise, car il porte une blouse tandis que les ouvriers sont en combinaison.

L'autocontrôle ambitionne de remplacer le jeu du chat et de la souris entre contrôleurs et contrôlés par une déclaration spontanée des défauts, dès leur apparition et quel qu'en soit le motif (pièce ou outil défectueux, maladresse...). Le système repose sur la fidélité des comptes rendus des monteurs ; pour éviter toute " dérive ", le moniteur de chaque section contrôle régulièrement un véhicule de fond en comble; ces " audits " sont réalisés à des heures tirées au sort par un programme informatique. Tous les agents de maîtrise et la plupart des cadres (directeur compris) " auditent " également de temps en temps. Si jamais l'un de ces contrôles révèle un défaut non signalé, le moniteur vérifie toutes les voitures montées depuis l'audit précédent et avertit le monteur de son oubli. Dans les cas graves, on sort le fautif de la chaîne pour lui montrer, en aval, les conséquences de son erreur : on proclame ainsi à la face de l'atelier qu'il ne joue pas le jeu.

Le long de la chaîne, chaque chef d'équipe affiche un tableau jaugeant ses subordonnés à l'aune du système : c'est un décompte journalier du nombre de défauts non signalés par chaque monteur en autocontrôle, qui indique aussi 
les noms - éventuellement soulignés en rouge - des trois « moins bons» ouvriers du mois précédent. Certains chefs d'équipe y ajoutent un récapitulatif annuel où l'œil remarque aisément trois petites taches rouges chaque mois. Comme le dit un technicien chargé de conseiller chefs d'équipes et moniteurs en matière d'autocontrôle : " C'est la transparence, on ne cache rien, tout le monde est au courant de tout le monde, tout le monde surveille tout le monde. " Le chef de service : «Au lieu de 20 chefs qui surveillent et 2000 qui travaillent, les 2000 s'autosurveillent. "

Alors que ce genre de " résultats qualité » permet de comparer les ouvriers entre eux et les équipes entre elles, un autre dispositif a été imaginé pour mettre en compétition les échelons supérieurs de l'échelle hiérarchique. Les niveaux supérieurs de la maîtrise et les cadres responsables d'unités budgétaires sont ainsi classés « en gestion favorable » ou " défavorable ». En fabrication, on est « favorable » si la production du mois dépasse un certain seuil (proportionnel au nombre d'ouvriers). A un niveau supérieur, cela dépend de l'utilisation du budget alloué. Chaque mois est publiée une liste des secteurs " en gestion favorable ". Honte à ceux qui n'y figurent pas, et qui ne seront pas non plus « en vert " sur un tableau récapitulatif disposé chez le chef de service. Un graphique similaire est mis en évidence dans le bureau du directeur de l'usine. Tous les responsables ont leurs objectifs - harmonisés avec un objectif global de $5 \%$ de gain de productivité -, sur lesquels ils rendent compte régulièrement.

Le nouveau pouvoir des ateliers en autocontrôle joue ainsi sur la compétition et sur la pression du groupe pour orienter l'activité des agents dans le sens des finalités de l'entreprise. Un autre exemple en est le " groupe ouvrier de gestion des congés ", chargé d'obtenir de ses pairs une répartition harmonieuse des jours d'absence pour ne pas gêner la production. L'autocontrôle se caractérise aussi par un souci marqué de cohérence entre niveaux hiérarchiques : les mêmes instruments d'évaluation (le schéma de gestion " favorable » ou « défavorable "), la même préoccupation pour la qualité (tous les hiérarques " auditent " parfois sur les chaînes), des contacts directs avec les grands chefs ; enfin, un même langage, prêché sans relâche par tous les chefs et centré sur la qualité des produits. Les revues internes abondent en articles qui la présentent comme « une question de survie ". C'est au nom de la qualité qu'on blâme. Ne dites plus « Je vous fous un avertissement ", dites " Je vous fait un rapport qualité » ou un " rapport de comportement ». Tous les mots-clés changent (sauf lapsus) dans la bouche de la hiérarchie : la chaîne devient la " ligne "; le pointeau (l'employé qui contrôle les fiches de pointage) est désormais " agent de relation »; l'agent de sécurité devient " animateur sécurité ».

La crainte du chômage constituait certes un puissant stimulant pour serrer les coudes autour de l'entreprise. L'industrie automobile était alors en crise ; la concurrence japonaise était mise en scène dans les articles techniques et dans les bandes dessinées des revues internes ("Qui relèvera le défi de la hyène de Tokyo ? ") ; nombre de membres du personnel étaient inquiets pour leur avenir et celui de leurs enfants. 
La comparaison entre les trois ateliers étudiés montre que les évolutions de la gestion s'accompagnaient de changements dans les lieux et les costumes. Le deuxième atelier était en autocontrôle depuis plusieurs années : les chefs d'équipe y disposaient de grands espaces où étaient présentées les performances de leur groupe ; les ouvriers portaient, à leur convenance, des combinaisons ou des blouses. En revanche, dans le troisième atelier, où il n'y avait ni autocontrôle ni compétition, le territoire du chef se limitait à deux armoires peu reluisantes en bord de chaîne, et la blouse était strictement réservée à la " maîtrise »: on ne disait pas «t'es passé chef d'équipe », mais " t'as eu ta blouse ». L'atelier où l'autocontrôle se mettait en place occupait à tous égards une position intermédiaire.

Dans l'atelier traditionnel, en somme, la blouse fait le chef, et la gestion " sur le terrain " s'effectue généralement sous forme d' " arrangements " locaux, opaques aux services centraux. Dans les ateliers en autocontrôle, en revanche, le chef ne se reconnaît pas de loin, et le système rend visibles et mesurables tous les individus des ateliers. Autrement dit, au lieu de la traditionnelle hiérarchie d'apparat (mais dépourvue d'instruments de consensus), la gestion moderne organise la visibilité des agents. Le chef peut bien alors partager sa blouse : son âme n'est plus dans sa vêture, mais dans les valeurs de l'entreprise, qu'il incarne. Au nom de la qualité, la compétition et la pression du groupe opèrent à tous les échelons, par le biais de techniques de visibilité, adaptées aux différents niveaux hiérarchiques ${ }^{26}$. Vu de l'extérieur, le système semble reposer sur une soudaine "motivation " des agents ; l'approche ethnologique montre comment se construit, grâce à l'articulation de pratiques symboliques et d'instruments de gestion, la légitimité des prêcheurs de cette nouvelle religion de la qualité.

Dans les ateliers en autocontrôle de l'entreprise étudiée, l'activité du personnel était orientée dans le sens des finalités officielles de l'organisation, d'une manière certes bien plus efficace que ne le faisait la mairie, mais tout n'y était pas parfaitement prévu pour autant. L'accent mis sur la compétition générait certaines critiques - neutralisées par l'invocation de la qualité - et certains effets inattendus. Par exemple, pour le relevé des "défauts non signalés " par chaque monteur, il y avait deux moyens d'être bien placé : soit faire peu d'erreurs, soit que les autres en fassent davantage. C'est pourquoi tel ouvrier, parfois, ne signalait plus à ses camarades les défauts qu'ils commettaient, même s'ils étaient rattrapables... Les chefs de service préparaient de nouveaux outils d'évaluation pour contrecarrer ces « effets pervers». Toutefois, ils ne cherchaient pas à prévoir le comportement des agents dans le détail ; au contraire, chacun se voyait reconnaître une marge de liberté (très restreinte - mais non nulle - en bas de l'échelle hiérarchique), à condition de mettre sa créativité au service de l'entreprise. La " transparence " de l'évaluation des uns et des autres, 
l'emboîtement des objectifs des différents niveaux hiérarchiques engageaient les protagonistes dans un jeu de rôles difficile à éluder, non plus sous l'œil du chef, mais sous l'œil de la Qualité : c'était un "système verrouillé ", selon l'expression qu'affectionnait un chef de service, qui exprimait ainsi l'ambition d'orienter de manière décisive les actions de ses subordonnés ${ }^{27}$.

On sait que le pouvoir fait toujours appel à une certaine transcendance ${ }^{28}$. Dans l'entreprise étudiée, les discours de motivation invoquaient la qualité et la productivité, et mettaient en scène la « survie » du groupe. Il n'était pas nécessaire d'adhérer totalement aux valeurs prêchées par la hiérarchie pour y être sensible ; chacun savait que ces fins étaient censées s'imposer à tous, et ceci leur donnait une considérable efficacité potentielle. La technologie de gestion s'articulait à leur invocation et forgeait des « ressorts de rappel » destinés à redresser les comportements déviants. En revanche, la mairie s'en remettait aux normes formelles pour inscrire les finalités de l'organisation dans le quotidien des agents ; aucun dispositif particulier n'était prévu pour hiérarchiser les valeurs susceptibles d'opérer dans le champ du palais (l'administration locale, la promotion du parti au pouvoir, le soin des familles des employés...) ; les agents devaient arbitrer par eux-mêmes selon les signes transmis par les différents réseaux.

Les finalités d'une institution ne se situent pas au même niveau d'abstraction que les faires et les dires de ses membres; elles transcendent en quelque sorte les protagonistes, forment un cadre d'interprétation qui donne un sens à leurs actes et légitime l'exercice de l'autorité29. Les normes se placent à un niveau intermédiaire : elles instrumentent les finalités, sont modifiées selon certaines procédures et sont censées régler plus ou moins précisément le comportement des agents. Des valeurs extérieures, définies au niveau de la société globale, peuvent aussi être invoquées ; les activités des membres d'une organisation sont donc analysables en termes d'actes de communication interprétables à plusieurs niveaux : tel employé prépare-t-il un dossier, exécute-t-il l'ordre de son chef, travaille-t-il pour le parti ou pour la patrie ? Il n'y a pas de réponse unique ; chacun construit une ou plusieurs significations en fonction de sa position et de ses informations.

L'action des individus et l'élaboration de leur identité sont orientées par le fait d'être là, ensemble, selon les règles et les finalités institutionnelles ${ }^{30}$. L'identité d'un membre se construit d'abord à partir de sa position dans l'ordre institué et des moyens qui en découlent (bureau, outils, possibilités de parole...), sans que ces éléments s'inscrivent d'une manière prédéterminée : chacun investit à sa façon son lieu et son temps dans l'organisation. L'agencement de son mobilier, de ses outils de travail, de son emploi du temps lui renvoie l'image identitaire de l'espace-temps subjectif qu'il se forge dans l'organisation ${ }^{31}$.

A partir d'une analyse ethnologique de la dynamique du vocabulaire d'adresse en entreprise, j'ai proposé dans un texte antérieur ${ }^{32}$ de caractériser l'organisation moderne comme ordre symbolique, vu la prégnance du principe de différenciation hiérarchique entre les membres. D'une manière plus générale, les grandes organisations de la société moderne semblent pouvoir être appréhendées 
comme ordres de communication. Ordres créés "sur le tas " ou ordres préconçus par des architectes spécialisés ${ }^{33}$, les médiations organisationnelles évoquent les traits caractéristiques du langage humain : par l'assemblage de symboles et l'élaboration d'oppositions significatives (préexistantes - clivages sexuels et générationnels - ou créées à l'intérieur de l'organisation - classifications hiérarchiques, distinctions entre services...), elles définissent une facette de l'identité de leurs membres et leur permettent de communiquer, tout en conditionnant par là-même leurs échanges et leurs modes de pensée. Bien entendu, les agents d'une organisation ne se cantonnent pas nécessairement aux rôles prévus pour eux : l'organisation « formelle " est à la fois contrainte et matériau manipulable ${ }^{34}$.

Un jeu de construction dont les pierres sont matérielles ou symboliques, un mode d'emploi réglementaire, voilà ce que l'organisation propose à ses membres. Elle se donne à voir comme un modèle de la pensée logique, car son découpage du réel se veut gouverné par la correspondance des fins et des moyens. Toutefois, selon les manières dont les " ordres de communication " organisationnels articulent les menus détails et les idées, les membres peuvent utiliser ou non les éléments de l'organisation à diverses fins, voire manipuler les finalités officielles au service d'autres desseins, ou y mobiliser - notamment par des pratiques symboliques - des facettes " extérieures » de leur identité. Outre les moyens de coercition dont dispose l'autorité officielle, les grandes organisations fournissent donc toute une panoplie d'instruments aux " pouvoirs subtils " foucaldiens, et ouvrent des " espaces-temps " spécifiques pour mobiliser des valeurs et articuler des réseaux. Les membres ont ainsi, chacun à leur niveau, des champs d'action normalisés et de vastes potentialités de " bricolages " matériels et symboliques ${ }^{35}$. Bien que la prégnance des médiations dans les organisations en fasse des groupes particulièrement " inauthentiques ", l'ethnologie peut y trouver des terrains prometteurs pour l'interprétation de la modernité, notamment par l'analyse des " ordres de communication » de la grande industrie.

Centre de Recherche en Gestion (École Polytechnique)

\& Laboratoire d'Anthropologie urbaine (CNRS), Paris

\section{NOTES}

1. Lévi-Strauss $1958: 400-401$.

2. L'intérêt croissant des ethnologues pour les sociétés industrielles (ou post-industrielles, selon la célèbre formule d'Alain Touraine) n'est pas né d'un renouveau théorique, mais a pris la forme d'un " glissement progressif " (DELAPORTE $1986: 155)$. Marc ABÉLĖs (1989: 338-341) a retracé brièvement cette évolution.

3. LÉvi-Strauss $1983: 12$.

4. Une " attitude intellectuelle qui se nourrit de connaissances théoriques et éventuellement d'une expérience de plusieurs terrains " (DELAPORTE $1987: 243$ ).

5. LEROI-GOURHAN $1968: 1817$. 
6. Selon les expressions de Marc Augé (1987: 25).

7. Le séminaire « Ethnographie des organisations ", qui s'est déroulé de 1983 à 1988 au Ministère de la Recherche, à l'initiative du Centre de recherche en gestion de l'École polytechnique, a réuni des chercheurs de différentes disciplines (ethnologie, sociologie, économie, gestion...) et des praticiens (ingénieurs, gestionnaires, consultants...) intéressés par une approche distanciée et détaillée du fonctionnement des organisations. La construction de l'étrangeté du chercheur et de sa « bonne distance " à l'objet, ainsi que la relation entre l'observateur et les observés, ont tenu une place importante dans les discussions. Voir «Ethnographie des organisations » (1986).

8. AuGÉ $1987: 24$.

9. LEROI-GOURHAN $1968: 1817$.

10. Dans ses Principles of Scientific Management (1911), l'ingénieur américain Frederick W. Taylor posa les bases d'un systèmes d'organisation séparant strictement la conception du travail et l'exécution, et dans lequel les temps d'exécution des tâches des ouvriers - considérés comme des rouages interchangeables - étaient rigoureusement contrôlés.

11. Voir Burawoy 1979, Holzberg \& Giovannini 1981 et Copans \& Bernier 1986 pour l'histoire de l'anthropologie industrielle.

12. Par ex. Gaudin, Calzada \& Reverchon 1989.

13. Parmi les publications récentes, voir par ex. FEnEYrol 1985 ; DAPHY 1988, 1991 ; SÉLim 1991 ; Magaud \& Sugrta 1990.

14. Une première version de l'étude de la mairie a été présentée au colloque Governo municipal na América Latina (Universidade Federal do Rio Grande do Sul, Porto Alegre, Brésil, décembre 1989).

15. L'Argentine est un pays fédéral divisé en 22 provinces. Les provinces sont subdivisées en départements. Trois niveaux d'organisation politique correspondent donc à la division du territoire : le gouvernement national, les gouvernements provinciaux et les gouvernements municipaux des départements. Les lois sont élaborées, soit par le Parlement national, soit par un Parlement provincial.

16. Le péronisme (du nom du général Juan Perón) domina la scène politique argentine durant plusieurs décennies où il fut tour à tour au pouvoir ou proscrit. C'est un « mouvement " dans lequel les syndicats jouent un rôle important. "Le projet de société qu'il propose est celui de la 'communauté organisée' où différentes forces sociales, patrons et ouvriers se concertent sous l'autorité paternelle de l'État pour orienter le développement du pays " (RouQuí 1984 : 93). Perón, élu président en 1946, fut chassé par un coup d'État militaire en 1955, puis réélu en 1973 après dix-sept ans d'exil. Il mourut un an plus tard, laissant le pouvoir à la vice-présidente, sa veuve, laquelle fut renversée par le coup d'État de 1976. Le parti péroniste perdit les premières élections qui suivirent le rétablissement de la démocratie, en 1983, puis un nouveau président péroniste, Carlos Menem, fut élu en 1989.

17. Le personnel municipal était divisé en quatre sous-groupes : ouvriers et administratifs (catégories 2 à 9), personnel hiérarchique (catégories 11 à 15), professionnels (catégories 6 à 17), personnel de direction (catégories 18 à 25). Le salaire d'un agent dépendait de sa catégorie et de son ancienneté $(+3 \%$ par an). Les coordinateurs percevaient des salaires différenciés, proches de certains niveaux " hiérarchiques " ou " de direction".

18. Catégorie par laquelle le discours péroniste identifie parfois ses ennemis.

19. BALANDIER $1985: 97$,

20. Par rapport aux principes et normes du système social, les pratiques sont à envisager sous l'angle de la conformité, de la stratégie, de la manipulation ou de la contestation (BALANDIER 1978 : XI).

21. Selon l'expression de Foucault (1975: 105), qui a souligné l'importance du contrôle des détails dans le fonctionnement des institutions disciplinaires.

22. La manipulation était avant tout le fait du groupe de la secrétaire mais les professionnels y avaient aussi recours : un professionnel avait ourdi un blocage des études pour porter préjudice au directeur ; un autre avait organisé avec ses alliés du palais le "pompage " des dossiers traités par le directeur, c'est-à-dire leur renvoi systématique d'un service à l'autre.

23. Voir Guigo 1991a pour une présentation générale de la mairie.

24. Par exemple, à l'occasion des conflits dans le service de l'urbanisme, étant donné que les trois supérieurs hiérarchiques du directeur étaient le maire, son fils (secrétaire de mairie) et sa future bellefille (sous-secrétaire), les professionnels échafaudèrent des plans d'action en fonction de l'état supposé des relations dans la famille dirigeante. Cette combinaison de réseaux rappelle la caractérisation de la vie urbaine selon Ulf HANNERZ (1983) : a network of networks. 
25. Un rapport interne a été remis à la direction du personnel, et un article, dont certains éléments vont être repris ici, est paru par ailleurs (Guigo 1987). Signalons qu'avant de m'orienter vers l'ethnologie, j'avais occupé pendant plusieurs années un poste d'ingénieur dans cette même entreprise.

26. Cette visibilité généralisée évoque l'analyse de Michel Foucault (1975) sur l'évolution des systèmes disciplinaires.

27. Lors d'un entretien avec un dirigeant d'une autre entreprise qui revendique un management participatif, mon interlocuteur a évoqué l'image de l'entonnoir, qui traduit une idée analogue à celle du système verrouillé, mais sous un jour moins inquiétant.

28. BALANDIER $1978: 46,119$.

29. Dans une perspective d'économie politique, BOLTANSKI \& THÉVENOT (1987) ont proposé d'analyser l'action collective sous l'angle de la confrontation de différents " principes supérieurs communs ", transcendant les personnes et revendiqués par les protagonistes.

30. Douglas (1987 : 128) écrit : «For better or worse, individuals really do share their thoughts and they do to some extent harmonize their preferences, and they have no other way to make the big decisions except within the scope of institutions they build ", mais n'utilise pas de matériaux ethnographiques appartenant à des organisations modernes.

31. «Les gens investissent un espace d'une identité symbolique [...], en retour, cet espace et son utilisation leur renvoient leurs propres images identitaires " (BRODY 1987 : 86). Voir FISHER 1980 pour une étude générale de l'appropriation de l'espace industriel.

32. GuIGo 1991b.

33. Pour reprendre la distinction établie par LÉvi-STRAUSs (1958: 347) entre " ordres vécus " et " ordres conçus ").

34. De nombreux travaux sociologiques ont souligné le fait que les membres d'une organisation ne se limitent pas à leur rôle formel (BLAU 1955; DALTON 1959 ; TOURAINE 1965 ; CROZIER 1971; BENDIX 1974). Par comparaison, la démarche ethnologique apparaît plus attentive aux pratiques symboliques et plus ouverte.

35. Chez l'homme moderne, la pensée scientifique coexiste avec le " bricolage " des concepts (LÉvISTrauss 1962). M. Douglas (1987: 66-67) a proposé d'étendre la notion de bricolage aux institutions modernes. Françoise ZONABEND, dans un ouvrage récent (1989:180), a montré que le personnel d'une usine de retraitement nucléaire utilisait de diverses manières le système de règles de sécurité, échafaudant des « pratiques imaginatives ", sortes de " techniques d'approvisionnement 》 de la modernité.

\section{B I B L I O G R A P H I E}

ABÉlès, Marc

1989 Jours tranquilles en 89. Ethnologie politique d'un département français. Paris, Odile Jacob.

Augé, Marc

1987 «Qui est l'autre? Un itinéraire anthropologique », L'Homme 103 : 7-26.

BALANDIER, Georges

1978 Anthropologie politique. Paris, PUF. (1 re éd. 1967.)

1985 Le Détour. Paris, Fayard.

BENDIX, Reinhard

1974 Work and Authority in Industry. Berkeley-Los Angeles-London, University of California Press.

BLAU, Peter

1955 The Dynamics of Bureaucracy. Chicago, University of Chicago Press. 
BOLTANSKI, Luc \& Laurent THÉVENOT

1987 Les Économies de la grandeur. Paris, PUF (« Cahiers du Centre d'études de l'emploi »).

BRODY, Jeanne

1987 " Le Quartier de la rue des Rosiers ou l'histoire d'un cheminement ", in Jacques GuTwIRTH \& Colette PÉtonNet, s. dir., Chemins de la ville. Enquêtes ethnologiques. Paris, Comité des travaux historiques et scientifiques : 85-102.

Burawoy, Michael

1979 "The Anthropology of Industrial Work », Annual Review of Anthropology 8 : 231-266. Palo Alto (California).

COPANS, Jean \& Bernard BERNIER

1986 Présentation du numéro « Travail, industries et classes ouvrières ", Anthropologie et Sociétés 10 : 1-9. Montréal (Québec), Université Laval.

CROZIER, Michel

1971 Le Phénomène bureaucratique. Paris, Seuil («Points »). (1 éd. 1963.)

Dalton, Melville

1959 Men Who Manage. New York, John Wiley \& Sons.

DAPHY, Éliane

1988 «Sonoriser l'Opéra : la technique invisible ", Vibrations 5 : 145-162. Toulouse, Privat.

1991 " Le P.O.P.B. : technique du spectacle, spectacle de la technique ", in Anne-Marie Gourdon, s. dir., Publics de masse et salles polyvalentes. Paris, Éditions du CNRS (« Les Arts du Spectacle ") : 127-146.

DELAPORTE, Yves

1986 "L'Objet et la méthode. Quelques réflexions autour d'une enquête d'ethnologie urbaine ", L'Homme 97-98: 155-169.

1987 "De la Distance à la distanciation. Enquête dans un milieu scientifique ", in Jacques Gut. WIRTH \& Colette PÉTONNET, s. dir., Chemins de la ville. Enquêtes ethnologiques. Paris, Comité des travaux historiques: 229-245.

DOUGLAS, Mary

1987 How Institutions Think. London, Routledge \& Kegan Paul. (1 ${ }^{\text {re }}$ éd. Syracuse, NY, Syracuse University Press, 1986).

« Ethnographie des organisations ", n spéc., Formation et Gestion, printemps 1986.

Ethnographie des organisations. Actes du séminaire du Centre de recherche en gestion de l'École polytechnique, 1984-1988.

Feneyrol, Nicole

1985 La Communication en procès. Essai d'ethnologie dans l'entreprise. Thèse de l'EHESS.

FISHER, Gustave-Nicolas

1980 Espace industriel et liberté. Paris, PUF.

Foucault, Michel

1975 Surveiller et punir. Paris, Gallimard (" Bibliothèque des Histoires »).

Gaudin, Pierre, Rémi Calzada \& Claire Reverchon

1989 L'Une fume, l'autre pas. Fondation Royaumont.

Guigo, Denis

1987 «L'Empire du consensus », Gérer et comprendre. Annales des Mines 6 : 19-26; 7 : 24-33.

1991a Poderes en la Administración Municipal. Buenos Aires, Centro Editor de América Latina (« Biblioteca Política Argentina »).

1991b «Les Termes d'adresse dans un bureau parisien », L'Homme 119: 43-61. 
HANNERZ, Ulf

1983 Explorer la ville. Éléments d'anthropologie urbaine. Paris, Éditions de Minuit (« Le Sens commun »). (Éd. origi. : Exploring the City, New York, Columbia University Press, 1980).

HOLZBERG, Carol \& Maureen GIOvanNiNI

1981 "Anthropology and Industry : Reappraisal and New Directions ", Annual Review of Anthropology $10: 317-360$. Palo Alto (California).

LeROI-Gourhan, André

1968 "L'Expérience ethnologique ", in Jean POIRIER, s. dir., Ethnologie générale. Paris, Gallimard ("La Pléiade ») : 1816-1825.

LÉvi-Strauss, Claude

1958 Anthropologie structurale. Paris, Plon.

1962 La Pensée sauvage. Paris, Plon.

1983 Le Regard éloigné. Paris, Plon.

MAGAUd, Jacques \& Kurumi Sugita

1990 Angers-Nagano. Une comparaison France-Japon. Paris, Rapport de recherche PIRTTEM-CNRSMRT.

POIRIER, Jean

1968 "Le Programme de l'ethnologie ", in Jean PoIRIER, s. dir., Ethnologie générale. Paris, Gallimard ("La Pléiade ») : 527-595.

RouQuí, Alain

1984 L'Argentine. Paris, PUF ("Que Sais-je ? ").

SÉlim, Monique

1991 L'Aventure d'une multinationale au Bangladesh. Ethnologie d'une entreprise. Paris, L'Harmattan.

TAYLOR, Frederick W.

1957 La Direction scientifique des entreprises. Paris-Verviers, Marabout. (Éd. orig. : The Principles of Scientific Management, 1911).

TOURAINE, Alain

1965 Sociologie de l'action. Paris, Seuil.

1969 La Sociologie post-industrielle. Paris, Denoël.

ZONABEND, Françoise

1989 La Presqu'île au nucléaire. Paris, Odile Jacob. 


\begin{abstract}
A B S T RACT
Denis GuIgo, Anthropological Perspectives in Modern Organization.-This paper presents an anthropological analysis of human behaviour in big modern organizations, based on two fieldworks in a French car company and an Argentinian municipality. A firm or a public office establishes a specific context for the activity of its members (work places, tools and regulations; agents' categorization and hierarchy...); these logics of differentiation-a peculiar cutting up of reality-are theoretically governed by an agreement concerning ends and means; the relative transcendency of official institutional objectives legitimates organizational authorities. Nevertheless, members may also invoke other facets of their identity and mobilize external principles of legitimacy; so they perform imaginative bricolages with the material and symbolic organizational elements, and mobilize the symbolic and the imaginary in their practices. Thus big organizations appear as promisory fields for anthropology; as in the study of " traditional " objects, the matter is not to apprehend the whole society, but « to distinguish levels which are worth comparing, and thus, significant " (Lévi-Strauss 1958: 100).
\end{abstract}

\title{
RESUMEN
}

Denis Guigo, Perspectivas antropológicas en organizationes modernas. - A partir de estudios de campo realizados en una empresa automotriz francesa y una municipalidad argentina, esta artículo presenta una caracterización antropólogica de la actividad humana en organizaciones modernas. Una empresa o administración proporciona el contexto de la actividad de sus miembros (lugares e instrumentos de trabajo, estatutos y jerarquización de los agentes...) ; estas lógicas de diferenciación del personal y repartición de tareas están teóricamente sometidas a un principio de correspondencia entre fines y medios; la trascendencia relativa de los fines oficiales de legitimidad a las autoridades institucionales. Sin embargo, los miembros - en tanto "seres totales ", según la expresión de Marcel Mauss - también pueden movilizar otras facetas de su identidad, y realizar un bricolage imaginativo con los elementos materiales o simbólicos de la organización. Asimismo se pone énfasis en las prácticas de movilización de lo simbólico y lo imaginario, por parte de miembros que ejercen, o no, la autoridad formal. Las grandes organizaciones entonces aparecen como campos interesantes para la antropología ; no se trata de conocer todo de los grupos investigados, sino de " discriminar niveles adecuados para la comparación, y por lo tanto, significativos" (Lévi-Strauss 1958 : 100). (Traduit par l'auteur.) 